\title{
Implicit incentives for fund managers with partial information
}

\author{
Flavio Angelini ${ }^{1} \cdot$ Katia Colaneri ${ }^{2} \cdot$ Stefano Herzel $^{2} \cdot$ Marco Nicolosi $^{1}$ ii
}

Received: 18 November 2020 / Accepted: 7 May 2021 / Published online: 27 May 2021

(c) The Author(s) 2021

\begin{abstract}
We study the optimal asset allocation problem for a fund manager whose compensation depends on the performance of her portfolio with respect to a benchmark. The objective of the manager is to maximise the expected utility of her final wealth. The manager observes the prices but not the values of the market price of risk that drives the expected returns. Estimates of the market price of risk get more precise as more observations are available. We formulate the problem as an optimization under partial information. The particular structure of the incentives makes the objective function not concave. Therefore, we solve the problem by combining the martingale method and a concavification procedure and we obtain the optimal wealth and the investment strategy. A numerical example shows the effect of learning on the optimal strategy.
\end{abstract}

Keywords Portfolio management · Optimal control $\cdot$ Learning

The research of F. Angelini and M. Nicolosi was supported by Ricerca di Base 2017-2019 grant from the University of Perugia. The work of K. Colaneri was partially supported by Indam-Gnampa through the Grant n. U-UFMBAZ-2020-000791. S. Herzel acknowledges partial supports from the project HiDEA (Advanced Econometric methods for High-frequency Data), financed by MIUR under the program PRIN-2017 Prot. 2017RSMPZZ.

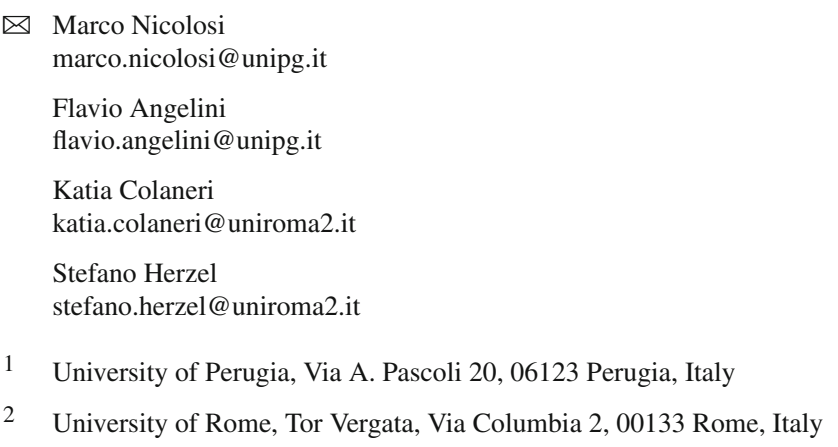




\section{Introduction}

The reward of a fund manager usually increases when the Asset Under Management (AUM) grows, while it decreases when the AUM shrinks. The AUM may grow either because of a higher value of the assets or because of new money flowing into the fund. Good performances of the fund with respect to its relative benchmark are likely to attract new investors. Therefore, contracts based on the AUM create an implicit incentive for the manager to beat the benchmark. We study the problem of a portfolio manager whose compensation depends on the AUM modelled through the relative performances with respect to a benchmark. This framework generalizes the setting of Basak et al. (2007), by considering a market model with one risk-free and one risky asset whose expected returns depend on an unobservable stochastic process, the "market price of risk". We introduce the realistic assumption that the manager has a limited knowledge on the market, she can only observe stock prices and estimates the market price of risk from them. Therefore, the manager is facing an optimization problem under partial information.

Optimization problems under partial information are usually solved in two steps: the first step, called reduction, consists of deriving the conditional distribution of the market price of risk with respect to the observed information flow; the second step solves the equivalent problem under the observed information. An important feature of our setting is that, while the market of claims contingent to the knowledge of the market price of risk is incomplete, the market restricted to those claims contingent only to stock prices is instead complete. We will exploit this fact to solve the optimization problem applying a martingale approach with the unique equivalent martingale measure (under the restricted setting) and then using a concavification argument to determine the unique optimal solution.

Although both problems of relative incentives and of optimization under partial information have been separately addressed in many papers (see the literature review provided in Sect. 1.1), this one is, to the best of our knowledge, the first contribution that analyses the combined effect of such issues on the optimal strategy of a portfolio manager. We contribute to the literature by providing the solution to the optimization problem in semi-closed form and we present one example where we show that the optimal strategy depends on the risk aversion of the manager and on the economic situation of the market. When the risk aversion of the manager is larger (lower) than that of a manager equipped with a logarithmic utility, she will tend to decrease (increase) her investment in the risky asset to hedge against the future adjustment in the estimates of the unknown parameter. We will also see that the larger the uncertainty on the value of the market price of risk, the greater the impact on the strategy and the consequent benefits in terms of expected utility when following the optimal strategy. Finally, as expected, the effect of learning becomes more tangible for longer time spans.

The paper is organized as follows. After a literature review (Sect. 1.1), Sect. 2 presents the market model and the portfolio optimization problem faced by the manager. In Sect. 3 we solve the optimization problem in two steps. First, we derive the dynamics of the filtered estimate of market price of risk, in order to reduce the problem to a common information flow. This procedure allows to obtain market dynamics driven by a unique source of randomness and hence the market model under partial 
information turns out to be complete. Second, we apply the martingale method along with concavification to characterize the optimal final wealth and the optimal investments strategy. Section 4 contains a numerical illustration of our results. Conclusions and comments are provided in Sect. 5. Proofs and computations are collected in the Appendix.

\subsection{Literature review}

The structure of portfolio managers' compensation is studied for instance in Ma et al. (2019), who show that performance based incentives represent the main form of compensation for portfolio managers in the US mutual fund industry. Of course, this is not the only type of incentive for fund managers. Option-like incentives of different nature (as for example management fees, investor's redemption options or funding options by prime brokers) apply in fund managers compensation contract, and influence manager's leverage decisions (see, e.g. Lan et al. 2013; Buraschi et al. 2014)

Basak et al. (2007) compute the optimal strategy followed by the manager under the assumption that she knows exactly the parameters driving the asset price process. They show that, when at an intermediate date the return of the fund is either very low or very large compared to the benchmark, the manager forgets about the implicit incentives determined by the fund-flows and reverts to the normal strategy, that is the one determined by Merton (1971). However, when the current return is closer to the benchmark, the manager tilts her strategy from the Merton level to try to beat the benchmark. Nicolosi et al. (2018) extend their framework to consider mean-reversion either in the market price of risk or in the volatility. Basak et al. (2008) introduce additional restrictions on the set of admissible strategies to contrast the tendency of managers to increase riskiness when their portfolio under-performs the benchmark, in order to align managers' scope to that of investors. The optimal allocation problem for institutional investors concerned about their performance with respect to a benchmark index is studied in Basak and Pavlova (2013). Their objective is to show how incentives influence the prices of the assets hold by institutional investors. In particular the authors found that, differently from standard investors, institutions tend to form portfolios of stocks that compose the benchmark index, they push up prices of stocks in the benchmark index by generating excess demand for index stocks and induce excess correlation among these stocks. Carpenter (2000) analyses the optimal investment problem of a risk adverse manager who is compensated with a call option on the asset under management. In this paper the market model is assumed to be complete and the non-concavity of the objective function is addressed by introducing a concavification argument and showing that the optimal solution takes values on a set where the original non-concave objective function is equal to the minimal concave function dominating it. An explicit solution to this problem in the Black-Scholes setting is provided in Nicolosi (2018) while Herzel and Nicolosi (2019) extend the solution to the case of mean-reverting processes. The impact of commonly observed incentive contracts on managers' decisions is also studied in Chen and Pennacchi (2009), where the authors found that for particular compensation structure, when a fund is performing poorly, the deviation from the benchmark portfolio is larger than in case of good performance. 
Other important contributions on the literature of delegated portfolio management problem include Cuoco and Kaniel (2011), who investigate the case where managers receive a direct compensation, related to the performance, from investors and discuss asset price implications in equilibrium. Different compensation schemes have been considered, for instance, in Barucci and Marazzina (2016) in a portfolio optimization problem for a manager who is remunerated through a High Water Mark incentive fee and a management fee and in Barucci et al. (2019) where a penalty on the remuneration is applied if the fund value falls below a fixed threshold, namely a minimum guarantee.

Optimal asset allocation under partial information has been widely studied in the literature. Brendle (2006) considered the optimal investment problem for a partially informed investor endowed with bounded CRRA preferences in a market model driven by an unobservable market price of risk via the HJB approach. Hata and Sheu (2018) also included consumption. A more general setting, not necessarily Markovian, has been analysed for instance in Björk et al. (2010) and Lindensjo (2016), under the assumption of market completeness. The optimization problem in these papers is solved using the Martingale approach.

The partial information case in a delegated portfolio management has been considered in the recent literature by Barucci and Marazzina (2015) in a slightly different setting compared to ours, where market is subject to two regimes, modelled via a continuous time two-state Markov chain and in Huang et al. (2012) where investment learning is studied under a Bayesian approach.

Other contributions in the case where prices are modelled as diffusions are Lakner (1995, 1998). Brennan (1998) and Xia (2001) study the effect of learning on the portfolio choices, and Colaneri et al. (2020) address the problem of computing the price that a partially informed investor would pay to access to a better information flow on the market price of risk. Investment problems in a market with cointegrated assets under partial information are studied in some recent works as for instance Lee and Papanicolaou (2016) and Altay et al. (2018, 2020).

\section{Market model and the portfolio optimization problem}

We fix a probability space $(\Omega, \mathcal{F}, \mathbf{P})$. Let $\mathbb{F}=\left\{\mathcal{F}_{t}, t \geq 0\right\}$ be a complete and right continuous filtration representing the global information. We consider a market model with one risky asset with price $S_{t}$, the stock, and one risk-free asset with price $B_{t}$. We assume that the price of the risk-free asset follows

$$
\frac{d B_{t}}{B_{t}}=r d t
$$

with the constant $r>0$ representing the constant interest rate. The risky asset price is modelled by a geometric diffusion

$$
\frac{d S_{t}}{S_{t}}=\mu_{t} d t+\sigma d Z_{t}^{S}
$$


where $Z_{t}^{S}$ is a one dimensional standard Brownian motion, $\sigma>0$ is the constant volatility and the drift is the process

$$
\mu_{t}=r+\sigma X_{t}
$$

which depends linearly on the market price of risk $X_{t}$. The process $X_{t}$ satisfies

$$
d X_{t}=-\lambda\left(X_{t}-\bar{X}\right) d t+\sigma_{X} d Z_{t}^{X}
$$

with starting value $X_{0}$ drawn from a normal distribution with mean $\pi_{0}$ and variance $R_{0}$. The parameter $\lambda>0$ is a constant representing the strength of attraction toward the long term expected mean $\bar{X}, \sigma_{X}>0$ is the volatility of the market price of risk and $Z_{t}^{X}$ is a one-dimensional standard Brownian motion correlated with $Z_{t}^{S}$ with correlation $\rho \in[-1,1]$. We assume that the market price of risk is a latent variable that is not directly observed, and its value can only be derived through the observation of $S_{t}$. That means that the available information is given by the filtration $\mathbb{F}^{S}:=\left\{\mathcal{F}_{t}^{S}, t \in[0, T]\right\}$, generated by the process $S .{ }^{1}$ Let us note that, since there are two risk factors $Z_{t}^{S}$ and $Z_{t}^{X}$, but only one traded asset besides the money market account, this market model is incomplete.

We study the problem of a fund manager who trades the two assets, $S_{t}$ and $B_{t}$, continuously in time on $[0, T]$, starting from an initial capital $w$. We assume that the stock does not pay dividends before time $T$. We describe the trading strategy of the manager by a process $\theta_{t}$ representing the fraction of wealth invested in the risky asset at any time $t \in[0, T]$. We only consider trading strategies that are self-financing and based on the available information, hence defining an admissible strategy as a selffinancing trading strategy, adapted to the filtration $\mathbb{F}^{S}$ and, to prevent arbitrage from doubling strategies, ${ }^{2}$ such that

$$
\mathbf{E}\left[\int_{0}^{T}\left(\left|\theta_{t} X_{t}\right|+\theta_{t}^{2}\right) d t\right]<\infty
$$

The set of all admissible strategies is denoted by $\mathcal{A}^{S}$. The wealth process generated by an admissible strategy $\theta_{t}$ is

$$
\frac{d W_{t}}{W_{t}}=\left(r+\theta_{t} \sigma X_{t}\right) d t+\theta_{t} \sigma d Z_{t}^{S}, \quad W_{0}=w>0 .
$$

The manager's compensation is determined by the value of the AUM at time $T$, expressed by $f\left(W_{T}, Y_{T}\right) W_{T}$, where $f$ is a function, to be defined below, that captures the effects of the relative performance with respect to the benchmark. The value of the

\footnotetext{
1 At any time $t, \mathcal{F}_{t}^{S}$ is the right continuous and complete $\sigma$-algebra generated by the process $S$ up to time $t$. Specifically, $\mathcal{F}_{t}^{S}:=\sigma\left\{S_{u}, 0 \leq u \leq t\right\} \vee \mathcal{O}$ where $\mathcal{O}$ is the collection of all $\mathbf{P}$-null sets. Notice that $\mathcal{F}_{t}^{S} \subset \mathcal{F}_{t}$, which models the fact the manager has a restricted information on the market.

2 Condition (5) is standard and prevents the wealth process to get unbounded. Weaker conditions on the set of admissible strategies can be considered, which may require a more technical approach.
} 
benchmark $Y_{t}$ is obtained by following the constant investment strategy $\beta$ and hence it follows

$$
\frac{d Y_{t}}{Y_{t}}=\left(r+\beta \sigma X_{t}\right) d t+\beta \sigma d Z_{t}^{S}
$$

The continuously compounded returns on the manager's portfolio and on the benchmark over the period $[0, t]$ are given by $R_{t}^{W}=\ln \frac{W_{t}}{W_{0}}$ and $R_{t}^{Y}=\ln \frac{Y_{t}}{Y_{0}}$, respectively. To compare relative performances, we set $Y_{0}=W_{0}$. The difference $R_{T}^{W}-R_{T}^{Y}$ provides the tracking error of the final wealth relative to the benchmark. The funds flow to relative performance relationship is described by the function

$$
f\left(W_{T}, Y_{T}\right)=\left\{\begin{array}{ccc}
f_{L} & \text { if } & R_{T}^{W}-R_{T}^{Y}<\eta_{L} \\
f_{L}+\psi \cdot\left(R_{T}^{W}-R_{T}^{Y}-\eta_{L}\right) & \text { if } & \eta_{L} \leq R_{T}^{W}-R_{T}^{Y}<\eta_{H} \\
f_{H}:=f_{L}+\psi \cdot\left(\eta_{H}-\eta_{L}\right) & \text { if } & R_{T}^{W}-R_{T}^{Y} \geq \eta_{H}
\end{array}\right.
$$

with $f_{L}>0, \psi>0$, and $\eta_{L} \leq \eta_{H}$ and it is illustrated in Fig. 1. This simplified structure of the funds flow to relative performance relationship, called in the literature collar type, shows that if the manager return is below the benchmark return of at least $\eta_{L}$ or above the benchmark return of at least $\eta_{H}$, the flow rate received by the fund is flat (with different rates $f_{L}<f_{H}$ ). When the relative performance, measured in terms of tracking error, is between $\eta_{L}$ and $\eta_{H}$, the flow function is a linear segment with a positive slope. The function $f$ also has two kinks when the difference $R_{T}^{W}-R_{T}^{Y}$ reaches the levels $\eta_{L}$ and $\eta_{H}$. The funds flow to relative performance relationship in Eq. (7) was proposed by Basak et al. (2007) to describe an implicit incentive scheme and it is based on the empirical analysis of Chevalier and Ellison (1997). The idea is that, if the fund under-performs with respect to the benchmark, investors tend to withdraw their money, the AUM decreases, and the manager receives a lower compensation. The opposite happens in the case of over-performance. Citing Basak et al. (2007): “(...) this simple way of modeling fund flows is able to capture most of the insights pertaining risk-taking incentives of a risk averse manager".

The manager maximizes the expected utility of her implicit incentives over the set of admissible strategies $\mathcal{A}^{S}$,

$$
\max _{\theta \in \mathcal{A}^{S}} \mathbf{E}\left[u\left(W_{T} f\left(W_{T}, Y_{T}\right)\right)\right]
$$

with initial budget $W_{0}=w$. We assume that the manager is endowed with a power utility function

$$
u(x)=\frac{1}{1-\gamma} x^{1-\gamma}
$$

with nonnegative risk aversion parameter $\gamma \neq 1$. The case $\gamma=1$ corresponds to the logarithmic utility. Since the market price of risk is not observable, this is an optimization problem under restricted information. To solve it, we first reduce it to 


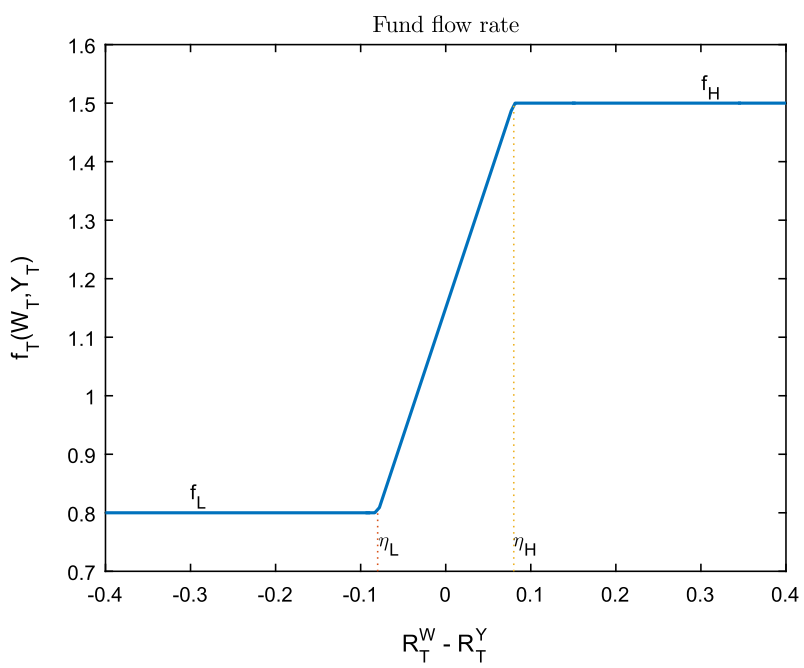

Fig. 1 The funds flow $f\left(W_{T}, Y_{T}\right)$ as a function of relative performance $R_{T}^{W}-R_{T}^{Y}$, with parameters $f_{L}=0.8, f_{H}=1.5, \eta_{L}=-0.08$ and $\eta_{H}=0.08$

a setting with a common information flow by replacing the unobservable process $X_{t}$ with its conditional expectation. This standard procedure allows us to consider an equivalent optimization problem under the available information, see, e.g. Fleming and Pardoux (1982). We characterize the conditional expectation of $X_{t}$ in the next section via Kalman filtering.

\section{Optimal wealth and strategies}

In this section we solve the problem (8). The first step is to estimate the unobservable market price from stock prices. Applying the Kalman filtering theory ${ }^{3}$ we get that the conditional distribution of market price of risk is Gaussian with conditional mean $\pi_{t}:=\mathbf{E}\left[X_{t} \mid \mathcal{F}_{t}^{S}\right]$, and conditional variance $R_{t}:=\mathbf{E}\left[\left(X_{t}-\mathbf{E}\left[X_{t} \mid \mathcal{F}_{t}^{S}\right]\right)^{2} \mid \mathcal{F}_{t}^{S}\right]$. To derive $\pi_{t}$ and $R_{t}$ we introduce the innovation process

$$
I_{t}:=Z_{t}^{S}+\int_{0}^{t}\left(X_{u}-\pi_{u}\right) d u .
$$

It is well known (see, e.g. Lipster and Shiryaev 2001 or Ceci and Colaneri 2012, 2014) that $I_{t}$ is a Brownian motion with respect to the observable filtration $\mathbb{F}^{S}$. The proposition below, proved for instance in Lipster and Shiryaev (2001), provides the dynamics of $\pi_{t}$ and $R_{t}$.

\footnotetext{
${ }^{3}$ Notice that the stock price process $S$ and its log-return generate the same type of information. This is a key feature since the drift of the log-return is a linear function of $X$, and hence the Kalman filter applies. The same setting has been considered for instance in Colaneri et al. (2020).
} 
Proposition 1 The conditional mean and variance of the market price of risk satisfy the equations

$$
\begin{array}{ll}
d \pi_{t}=-\lambda\left(\pi_{t}-\bar{X}\right) d t+\left(R_{t}+\rho \sigma_{X}\right) d I_{t}, & \pi_{0} \in \mathbb{R}, \\
d R_{t}=\left[\sigma_{X}^{2}-2 \lambda R_{t}-\left(R_{t}+\rho \sigma_{X}\right)^{2}\right] d t, & R_{0} \in \mathbb{R}^{+} .
\end{array}
$$

From (12) we see that the conditional variance of the market price of risk is deterministic and satisfies a Riccati ordinary differential equation. Using Eq. (10), we get the dynamics of the stock, of the wealth process and of the benchmark with respect to the innovation process

$$
\begin{aligned}
\frac{d S_{t}}{S_{t}} & =\left(r+\sigma \pi_{t}\right) d t+\sigma d I_{t}, \\
\frac{d W_{t}}{W_{t}} & =\left(r+\theta_{t} \sigma \pi_{t}\right) d t+\theta_{t} \sigma d I_{t}, \\
\frac{d Y_{t}}{Y_{t}} & =\left(r+\beta \sigma \pi_{t}\right) d t+\beta \sigma d I_{t} .
\end{aligned}
$$

Since all processes are only driven by the innovation, the sub-market restricted to claims that can be replicated by strategies in $\mathcal{A}^{S}$ is complete. We solve the optimization problem (8) using the martingale method (see, for instance Cox and Huang 1989), transforming the dynamic optimization problem (8) where the control variable is a strategy, into an equivalent static problem where the control variable is the terminal wealth.

To identify terminal wealths that are reachable from the initial budget $w$ with feasible strategies, we introduce the unique state price density process

$$
\frac{d \xi_{t}}{\xi_{t}}=-r d t-\pi_{t} d I_{t}, \quad \xi_{0}=1 .
$$

The static optimization problem, equivalent to (8) is

$$
\max _{W_{T}} \mathbf{E}\left[u\left(W_{T} f\left(W_{T}, Y_{T}\right)\right)\right],
$$

with budget constraint

$$
w=\mathbf{E}\left[\xi_{T} W_{T}\right]
$$

The objective function in problem (17)-(18) is not concave in $W_{T}$. To overcome this issue we apply the concavification procedure proposed by Carpenter (2000) (see Appendix D for more details on the procedure). Following the approach in Proposition 2 of Basak et al. (2007), we define the optimal final wealth relative to the benchmark, which is given by $V_{T}=\frac{W_{T}^{\star}}{Y_{T}}$, where $W_{T}^{\star}$ is the optimal final wealth in problem (17)-(18). This quantity has an explicit representation (e.g. equation $(A 7)$ in Basak et al. 2007 or equation (6) in Nicolosi et al. 2018) given, for completeness, by 
Eq. (26) in Appendix A. One key characteristic is that $V_{T}$ is a function of $\zeta_{T}:=\xi_{T} Y_{T}^{\gamma}$ only. Computing $V_{T}$, enables us to characterize the optimal terminal wealth. However this is not sufficient to obtain the trading optimal portfolio strategy, for which, we need to know the value of optimal wealth at any time $t \in[0, T]$, and consequently, we must determine the relative wealth $V_{t}=\frac{W_{t}^{\star}}{Y_{t}}$. To compute $V_{t}$ we consider the benchmarked market, where we discount all processes with the numéraire $Y_{t} .{ }^{4}$ Due to market completeness there exists an equivalent risk neutral probability measure $\mathbf{Q}$ for the benchmarked market. Put in other words there exists a probability measure $\mathbf{Q}$ which is equivalent to $\mathbf{P}$ and such that the price process of any benchmarked traded asset (i.e. any traded asset discounted with $Y_{t}$ ) is a martingale under $\mathbf{Q}{ }^{5}$

We define the process $\zeta_{t}=\xi_{t} Y_{t}^{\gamma}$ and derive its distribution under $\mathbf{Q}$. Let $z$ be a complex number and define the conditional moment generating function of $\ln \left(\zeta_{T}\right)$ under the measure $\mathbf{Q}$ as

$$
H(t, \zeta, p ; z)=\mathbf{E}^{\mathbf{Q}}\left[\zeta_{T}^{z} \mid \mathcal{F}_{t}^{S}\right]=\mathbf{E}^{\mathbf{Q}}\left[\zeta_{T}^{z} \mid \zeta_{t}=\zeta, \pi_{t}=p\right]
$$

The function $H(t, \zeta, p ; z)$ plays a key role in solving the optimization problem (see Proposition 2 below). It is characterized in the following technical lemma.

Lemma 1 Let $z$ be a complex number and let $A(t ; z), B(t ; z)$ and $C(t ; z)$ be the solutions of the system of Riccati equations (40)-(41)-(42) in Appendix B on the interval [0,T]. Under suitable regularity conditions (see Eq. (35) in Appendix B), the conditional moment generating function of $\ln \left(\zeta_{T}\right)$ under the measure $\mathbf{Q}$ is given by

$$
H(t, \zeta, p ; z)=\zeta^{z} e^{A(t ; z)+B(t ; z) p+\frac{1}{2} C(t ; z) p^{2}} .
$$

The proof of Lemma 1 goes along the same lines as in Nicolosi et al. (2018). We remark that in this particular case, since both the drift and volatility in the dynamics of the filter $\pi_{t}$ are not constant (11), the coefficients of the Riccati equations that characterize the functions $A, B$ and $C$ are time-dependent. The solution of non-homogeneous Riccati equations are discussed in Appendix C.

In the next step we use Fourier Transform to compute the optimal relative wealth and the optimal strategy at any time $t \leq T$.

Proposition 2 Let $K_{j}$, for $j=1,2,3,4$ be real numbers such that $K_{1}<-1 / \gamma$, $K_{4}>-1 / \gamma$ and

$$
H\left(t, \zeta, p ; K_{j}\right)=\mathbf{E}^{\mathbf{Q}}\left[e^{K_{j} \ln \left(\zeta_{T}\right)} \mid \zeta_{t}=\zeta, \pi_{t}=p\right]<\infty
$$

Then,

\footnotetext{
4 Notice that the benchmark is a positive self-financing portfolio, and hence it can be taken as numéraire.

5 Introducing the measure $\mathbf{Q}$ allows us to circumvent technical difficulties: for instance, to get the optimal wealth $W_{t}^{\star}$ under the physical measure $\mathbf{P}$, one should know the joint distribution of $Y^{\gamma}$ and $\xi$. This is unnecessary if we perform the change of measure, where one can use the martingale property and get the distribution of $W_{t}^{\star}$ more directly. See, e.g. Proposition 2 in Basak et al. (2007).
} 
(i) the relative wealth $V_{t}$ is finite and given by

$$
V_{t}=\frac{1}{2 \pi} \sum_{j=1}^{4} \int_{-\infty}^{+\infty} \hat{\varphi}_{j}\left(u+i K_{j}\right) H\left(t, \zeta, p ; K_{j}-i u\right) d u
$$

where the functions $\hat{\varphi}_{j}(z)$ of the complex variable $z$ are given in Appendix A;

(ii) the optimal strategy is

$$
\theta_{t}=\beta+\frac{1}{\sigma V_{t}}\left(\frac{\partial V}{\partial \zeta} \zeta_{t}\left(\gamma \beta \sigma-\pi_{t}\right)+\frac{\partial V}{\partial p}\left(R_{t}+\rho \sigma_{X}\right)\right)
$$

The proof of Proposition 2 is given in "Appendix B". Notice that there are no conditions on constants $K_{2}$ and $K_{3}$, and for instance, if they are both equal to zero condition (20) is trivially satisfied. See the proof in Appendix B for further details. Examples of applications of the formulas for the optimal relative wealth (Eq. 21) and the optimal strategy (Eq. 22) provided by Proposition 2 are given in the next section.

\section{A numerical illustration}

Now we study the impact of uncertainty on the market price of risk on optimal strategies of a portfolio manager subject to implicit incentives. First we examine the combined effect of risk aversion and of market conditions, then we show that its relevance increases with the level of uncertainty and with the time span of the investment. In a relatively stable market (i.e. low volatility) with lower expected returns, the portfolio manager increases her exposure to the risky asset when underperforming and decreases it when overperforming. The opposite happens when the market is more volatile and expected returns are higher. Moreover, we see that risk-aversion has a direct influence on the views of the manager about the uncertain estimates. Managers with a riskaversion parameter larger than 1 fear that the value of the market price of risk will turn out to be below their current estimate and consequently reduce their exposure to hedge for the future changes. Managers with risk aversion lower than 1 expect that the value may be higher than the estimate and tilt their strategy in the opposite way.

To illustrate such behavior with an example, we consider a simplified version of our model, where the market price of risk is constant but unknown, and given by a random variable $X_{0}$, drawn from a Gaussian distribution with mean $\pi_{0}$ and variance $R_{0}$. This setting is analogous to that of Brennan (1998), where the manager does not know the value of the drift of the price process and can only estimate its expected value $m_{0}$, which is related to the market price of risk $\pi_{0}$ by

$$
\pi_{0}=\frac{m_{0}-r}{\sigma} .
$$


Setting $\lambda=0$ and $\sigma_{X}=0$, we get that the conditional expected value and variance follow

$$
d \pi_{t}=R_{t} d I_{t}, \quad \pi_{0} \in \mathbb{R},
$$

and

$$
R_{t}=\frac{R_{0}}{R_{0} t+1}
$$

To highlight the effects of uncertainty on the market price of risk we also consider a manager who erroneously assumes that $X_{0}=\pi_{0}$. We call this manager myopic because, unlike the far-sighting manager, she does not adjust her strategy to hedge for future changes on the estimates. We denote by $\theta_{t}^{0}$ the fraction of wealth invested by the myopic manager in the risky asset and by $\theta_{t}$ the optimal strategy of the partially informed manager given by Eq. (22). The value of $\theta_{t}^{0}$ is obtained from Eq. (22), by setting $R_{t}=0$ and $\pi_{t}=\pi_{0}$ (of course, this also affects the relative wealth $V_{t}$ ). As a comparison we also consider the Merton level $\theta_{N}=\frac{1}{\gamma} \frac{\mu_{0}-r}{\sigma^{2}}$ corresponding to the optimal investment of the myopic manager who optimizes only the utility of terminal wealth, without other incentives.

Figure 2 represents the myopic strategy $\theta_{t}^{0}$ (dotted line) and the optimal strategy under partial information $\theta_{t}$ (continuous line) as functions of the relative return of the portfolio with respect to the benchmark, that is $R_{t}^{W}-R_{t}^{Y}$, at time $t=0.25$, either for $\gamma=0.8$ (left panels) or for $\gamma=2$ (right panels). The parameters of the implicit incentives structure at time $T=1$ in Eq. (7) are the same as in Fig. 1.

The top panels show the case when the Merton level is above the investment in the risky asset of the benchmark portfolio, that is when $\theta_{N}>\beta$. In the illustration we set $\beta=1$. This setting corresponds to a market situation with relatively small volatility and returns, that is called economy (a) by Basak et al. (2007), obtained by taking $\sigma=0.15, r=0$ and $m_{0}=0.1$ in our model.

The bottom panels show the situation when the Merton level is below the investment in the risky asset of the benchmark, that is when $\theta_{N}<\beta$. This is the economy (b) in Basak et al. (2007), a more volatile and remunerative market, obtained by setting $\sigma=1, r=0$ and $m_{0}=0.3$. The level of uncertainty on the initial estimate is given by $R_{0}=0.09$ for all the panels.

The panels on the left represent a less risk averse manager $\gamma=0.8$, those on the right a more risk-averse one $\gamma=2$. By comparing the left to the right panels we see that the investment in risky asset decreases with risk aversion for both economies (a) and (b). The strategies for a myopic and a far-sighting manager are qualitatively similar to each other with the far-sighting manager taking either more or less risky positions than the myopic one, depending on the risk-aversion. When the risk aversion parameter $\gamma$ is equal to 1 (that is the case of logarithmic utility), the two strategies coincide. A far-sighting manager with a risk aversion smaller than 1 (left panels) tends to be more exposed to the risky asset than a myopic manager with the same risk aversion. In this case, the far-sighting manager acts optimistically, as she believes that, increasing the precision of the estimates of the market price, the correct value will be higher than the 

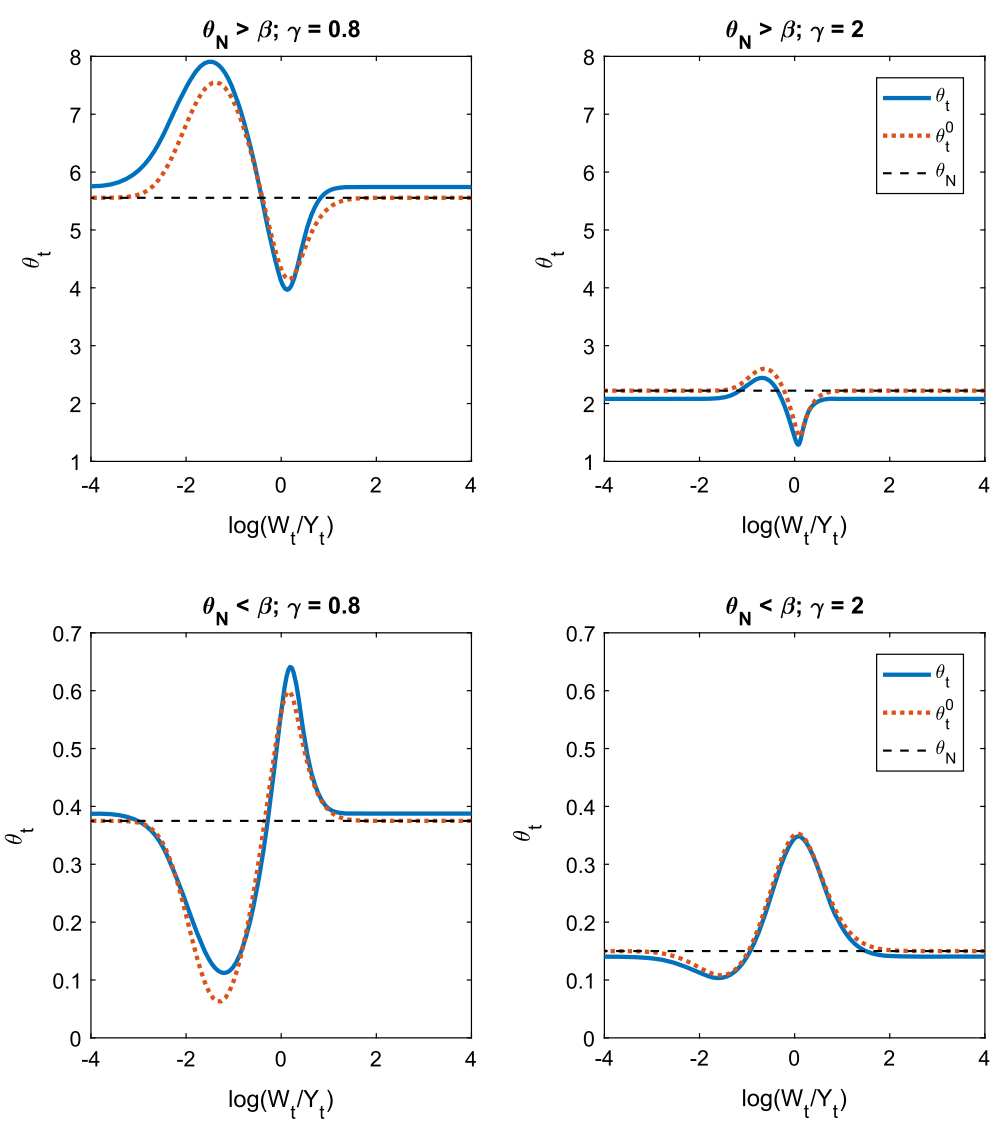

Fig. 2 Optimal strategies for different economies and different managers. The optimal strategy $\theta_{t}$ (continuous line) and the myopic one $\theta_{t}^{0}$ (dotted line) at time $t=0.25$ are reported as functions of the relative return of the portfolio with respect to the benchmark, as well as the Merton level $\theta_{N}$ (dashed line). Left panels represent the strategies of managers with risk aversion $\gamma=0.8$, right panels those of more risk-averse managers $(\gamma=2)$. Top panels represent economy a $\left(\theta_{N}>\beta=1\right)$, that is a less volatile market with higher returns $\left(\sigma=0.15, \pi_{t}=0.667\right)$, the bottom panels are referred to economy $\mathbf{b}\left(\theta_{N}<\beta=1\right)$, a more volatile market with lower returns $\left(\sigma=1, \pi_{t}=0.3\right)$. The parameters of the payoff function are the same as in Fig. 1. The others parameters are $T=1, r=0$, and $R_{0}=0.09$ so that the variance $R_{t}$ is 0.088 according to (24)

current one. Instead, the more risk-averse manager (right panels) is pessimistic and reduces the exposition to the risky asset fearing that the future estimates will be lower than the current one. By comparing top to bottom panels in Fig. 2 we see the effects of the overall economic condition on the optimal strategy, depending on the current results of the portfolio management strategy. When the relative performance is either too low or too high for the incentives to have an effect on the final reward, the optimal strategy approaches a constant level that corresponds to the optimal risky exposure without incentives and hence the myopic investment converges to the Merton level. If the manager is underperforming but still hopes to recover, or if she is slightly ahead, but still fearing to end behind, she adjusts the portfolio strategy in a way that depends 

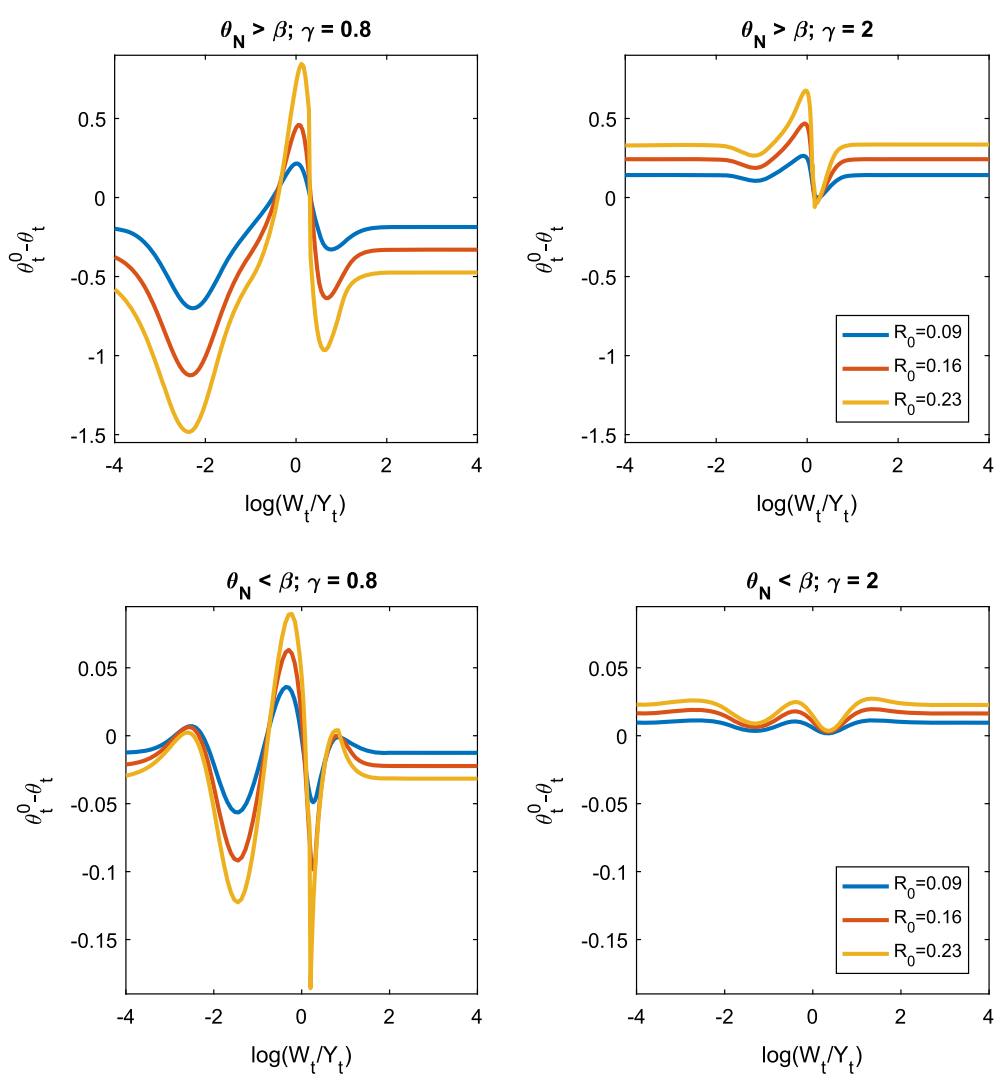

Fig. 3 Deviations $\theta_{t}^{0}-\theta_{t}$, at time $t=0.25$, as functions of the relative return of the portfolio with respect to the benchmark, for different levels of initial conditional variance $R_{0}=[0.09,0.16,0.23]$. Left panels represent the strategies of managers with risk aversion $\gamma=0.8$, right panels those of more risk-averse managers $(\gamma=2)$. Top panels represent economy a $\left(\theta_{N}>\beta=1\right)$, that is a less volatile market with higher returns ( $\left.\sigma=0.15, \pi_{t}=0.667\right)$, the bottom panels are referred to economy $\mathbf{b}\left(\theta_{N}<\beta=1\right)$, a more volatile market with lower returns $\left(\sigma=1, \pi_{t}=0.3\right)$. The parameters of the payoff function are the same as in Fig. 1. The others parameters are $T=1, r=0$

on the economic conditions. In the case of economy (a) (top panels), she increases the exposure when trailing and decreases it when leading. The economy (b), representing a more volatile market and higher expected returns (bottom panels), induces the same manager to take opposite choices.

Figure 3 represents, for the same scenarios in Fig. 2, the difference $\theta_{t}^{0}-\theta_{t}$, at time $t=0.25$, as a function of the relative return of the portfolios with respect to the benchmark, for some values of $R_{0}$. We can clearly see that the larger the values of $R_{0}$ the larger the differences, in absolute values, between the strategies.

To assess the impact of learning on the expected utility, we compute the Certainty Equivalent of the terminal wealth $W_{T}$, that is

$$
C E\left(W_{T}\right):=u^{-1}\left(E\left(u\left(W_{T}\right)\right)\right)
$$




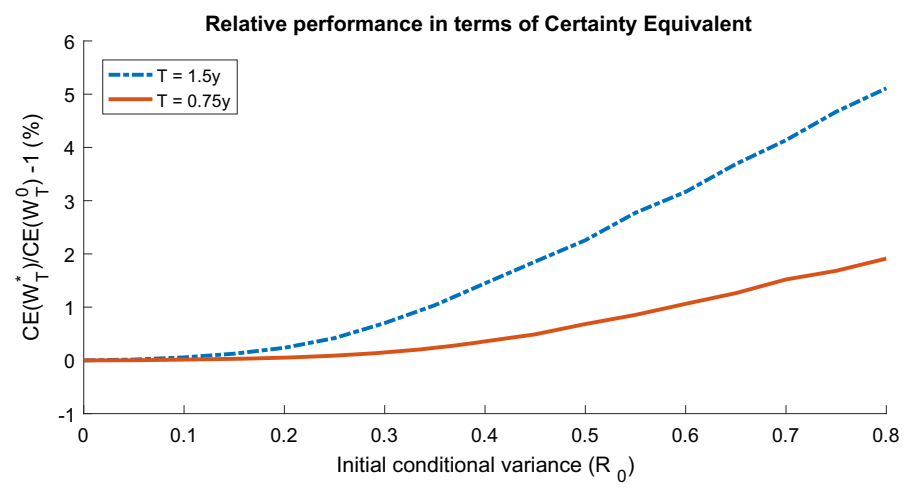

Fig. 4 The impact of learning on the relative increment of the Certainty Equivalent as a function of the initial variance $R_{0}$, for maturities 0.75 and 1.5 years. The other parameters are $\gamma=1.5, \sigma=0.15, \pi_{0}=0.667$

and the Relative Increment

$$
R I:=\frac{C E\left(W_{T}^{*}\right)}{C E\left(W_{T}^{0}\right)}-1
$$

where $W_{T}^{*}$ and $W_{T}^{0}$ are the terminal wealths reached by the optimal strategy and by the myopic one. Therefore, $R I$ measures the relative improvement of the Certainty Equivalent when adopting the optimal, far-sighting, strategy instead of the myopic one. To compute the terminal wealths we proceed by simulation, drawing first $X_{0}$ from a normal random variable with mean $\pi_{0}$ and variance $R_{0}$, and simulating the risky asset $S$. Then, we get $W_{T}^{*}$ and $W_{T}^{0}$ from Eq. (26). The expected utilities are obtained from the sample mean of the terminal utilities.

Figure 4 shows the values of $R I$ as a function of the variance of the market price of risk $R_{0}$, for two values of $T$. We see that the relative performance improves as $R_{0}$ increases and it is more pronounced for longer maturities. This is due to the fact that, while the far-sighting manager reacts to changes in market conditions by observing the asset prices and improves her estimates of the market price of risk, the myopic manager believes that the characteristics of the market are stable and never updates her estimates. Therefore, the two strategies are equal only in the case of zero uncertainty on the value of the market price of risk (when $R_{0}=0$ ), and their difference grows as $R_{0}$ and $T$ increase.

\section{Conclusions}

We studied a portfolio optimization problem for a manager who is compensated according to the performance of her portfolio relative to a benchmark. The manager invests in a risk-free asset and in a risky asset whose return depends on a latent variable representing the market price of risk. Hence she solves an optimization problem under partial information. Due to the implicit incentives given by the funds flow to relative performance, the utility function of the manager is not concave and hence 
existence of the optimum does not trivially hold. We solve the optimization problem using the martingale approach and a concavification procedure. This approach can be successfully applied due to completeness of the market under partial information. Optimal wealth and consequently the optimal strategy are characterized in a semiexplicit form via Fourier transform. We illustrated our results with an example, where we assume that the market price of risk is constant but unknown. We observed that the level of risk aversion has an influence on the manager's estimate of the market conditions, and consequently on her investment choices. Managers with a small risk aversion parameter are optimistic: they tend to increase their investment in the risky assets compared to myopic managers, believing that the true value of the market price of risk (and hence of the asset return) is more favourable than her estimate. It is also seen that if the market is not subject to large fluctuations, managers invest more in risky assets when they are underperforming the benchmark, in anticipation to retrieve benchmark revenues, and invest less in the risky asset when overperforming, to avoid possible downward movements of the market.

We have also analyzed the effect of uncertainty (i.e. $R_{0}$ ) on the optimal portfolio strategies and the optimal wealth by comparing the case of the myopic manager with the optimal partially informed investor. We have seen that the deviation between strategies amplifies as $R_{0}$ increases. The comparison between Certainty Equivalents corresponding to the optimal wealth (under partial information) and the wealth of the myopic manager, goes in the same direction, that is, it grows with $R_{0}$, and increases with time as well, as a consequence of the learning effect.

We conclude with a few clarifications on the setting under partial information and possible extensions. In this paper we assumed that the volatility is constant to end up in the setting of Kalman filtering. This is convenient for the optimization problems since in this case filter is finite dimensional. We also stress that if the volatility depends on the market price of risk (i.e. is a bijective function $\sigma\left(X_{t}\right)$ ) then we end up with a model under full information (see, e.g. Ceci and Colaneri 2017, Remark 3). Indeed, the volatility is always adapted to the filtration $\mathbb{F}$ and hence observable, therefore $X$ can be derived by function inversion. In this paper we have analyzed the optimal asset allocation under implicit incentives for the manager in the univariate case under partial information. The multivariate case is discussed under full information in Basak et al. (2007) with a constant market price of risk and Nicolosi et al. (2018) with a stochastic market price of risk. The generalization of our partial information problem to the multivariate case can be carried following the same steps of this paper; our choice of considering the one dimensional case, however, allows us to avoid technicalities and keep the notation simpler. For instance the multivariate case requires to filter the multidimensional market price of risk using the information coming from multiple risky assets. It is well known that, in such case, the conditional variance is characterized via a system of Riccati equations that does not have an explicit solution. We considered collar-type incentives, but our approach can be applied to study other incentive functions. Of course, a different incentive function will change the concavified objective 
function and, as a consequence, the functional form of the optimal wealth and the optimal strategies.

Funding Open access funding provided by Universitá degli Studi di Perugia within the CRUI-CARE Agreement.

Open Access This article is licensed under a Creative Commons Attribution 4.0 International License, which permits use, sharing, adaptation, distribution and reproduction in any medium or format, as long as you give appropriate credit to the original author(s) and the source, provide a link to the Creative Commons licence, and indicate if changes were made. The images or other third party material in this article are included in the article's Creative Commons licence, unless indicated otherwise in a credit line to the material. If material is not included in the article's Creative Commons licence and your intended use is not permitted by statutory regulation or exceeds the permitted use, you will need to obtain permission directly from the copyright holder. To view a copy of this licence, visit http://creativecommons.org/licenses/by/4.0/.

\section{A Optimal final wealth}

In this section we characterize the final wealth relative to the benchmark $V_{t}$, for every $t \in[0, T]$. We first consider the optimal final wealth relative to the benchmark, given by the random variable $V_{T}=\frac{W_{T}^{*}}{Y_{T}}$. Its expression has been computed in Basak et al. (2007) and also reported in Nicolosi et al. (2018), and in our framework, is given by

$$
V_{T}=\varphi_{1}\left(\zeta_{T} ; c_{1}\right)+\varphi_{2}\left(\zeta_{T} ; c_{1}, c_{2}\right)+\varphi_{3}\left(\zeta_{T} ; c_{2}, c_{3}\right)+\varphi_{4}\left(\zeta_{T} ; c_{3}\right)
$$

where $\zeta_{T}=\xi_{T} Y_{T}^{\gamma}$ and functions $\varphi_{j}$, for $j=1, \ldots, 4$ are

$$
\begin{aligned}
& \varphi_{1}\left(\zeta ; c_{1}\right)=f_{H}^{1 / \gamma-1} y^{-1 / \gamma} \zeta^{-1 / \gamma} \mathbf{1}_{\zeta<c_{1}} \\
& \varphi_{2}\left(\zeta ; c_{1}, c_{2}\right)=e^{\eta_{H}} \mathbf{1}_{c_{1} \leq \zeta<c_{2}} \\
& \varphi_{3}\left(\zeta ; c_{2}, c_{3}\right)=h(\zeta) \mathbf{1}_{c_{2} \leq \zeta<c_{3}} \\
& \varphi_{4}\left(\zeta ; c_{3}\right)=f_{L}^{1 / \gamma-1} y^{-1 / \gamma} \zeta^{-1 / \gamma} \mathbf{1}_{\zeta \geq c_{3}} .
\end{aligned}
$$

The value $y \in \mathbb{R}$ is the Lagrange multiplier that ensures that the budget constraint of the optimization problem $\mathrm{w}=\mathbf{E}\left[\xi_{T} W_{T}^{*}\right]$ is satisfied; the function $h(\zeta)$ is the solution of the equation

$$
\frac{d}{d v} u\left(v f_{L}+v \psi\left(\ln v-\eta_{L}\right)\right)=y \zeta
$$

Parameters $c_{1}, c_{2}$ and $c_{3}$, and hence the value of $V_{T}$, depend on the following relation, called Condition $A$ :

$$
\frac{\gamma}{1-\gamma}\left(\frac{f_{H}+\psi}{f_{L}}\right)^{1-1 / \gamma}+\left(\frac{f_{H}+\psi}{f_{H}}\right)-\frac{1}{1-\gamma} \geq 0
$$

This condition is related to concavification as explained in Appendix D. 
Proposition 2 of Basak et al. (2007) shows that, if Condition $A$ holds, then $c_{1}, c_{2}$ and $c_{3}$ in (26) satisfy $c_{1}=f_{H}^{1-\gamma} e^{-\gamma \eta_{H}} / y$, and $c_{3}=c_{2}>c_{1}$ satisfying $g\left(c_{3}\right)=0$, with

$$
g(\zeta)=\left(\gamma\left(\frac{y}{f_{L}} \zeta\right)^{1-1 / \gamma}-\left(f_{H} e^{\eta_{H}}\right)^{1-\gamma}\right) /(1-\gamma)+e^{\eta_{H}} y \zeta
$$

Hence, in this case, $\varphi_{3}\left(\zeta ; c_{2}, c_{3}\right)$ is the indicator function of the empty set and therefore it is zero.

When Condition $A$ is not met, Basak et al. (2007) show in Appendix $\mathrm{C}$ that $c_{1}=$ $f_{H}^{1-\gamma} e^{-\gamma \eta_{H}} / y, c_{2}=\left(e^{\eta_{H}} f_{H}\right)^{-\gamma}\left(f_{H}+\psi\right) / y$ and $c_{3}=\left(f_{L} \underline{V}\right)^{-\gamma} f_{L} / y$ with $\underline{V}$ being the left boundary of the region where the objective function is not concave. Denoting with $\bar{V}$ the right boundary of the non concave region, $\underline{V}$ and $\bar{V}$ can be computed as the points where the straight line between these two points is tangent to the objective function.

Next, we provide a representation for the function $\hat{\varphi}_{j}(z), j=1, \ldots, 4$, which are used to compute the optimal relative wealth $V_{t}$ given in Proposition 2 . The functions $\hat{\varphi}_{j}(z), j=1, \ldots, 4$, are the Fourier transforms of the functions in (27)-(28)-(29)-(30) and they are given by

$$
\begin{aligned}
& \hat{\varphi}_{1}(z)=f_{H}^{1 / \gamma-1} y^{-1 / \gamma} \frac{\left(c_{1}\right)^{-1 / \gamma+i z}}{-1 / \gamma+i z} \\
& \hat{\varphi}_{2}(z)=e^{\eta_{H}} \frac{\left(c_{2}\right)^{i z}-\left(c_{1}\right)^{i z}}{i z} \\
& \hat{\varphi}_{4}(z)=f_{L}^{1 / \gamma-1} y^{-1 / \gamma} \frac{\left(c_{3}\right)^{-1 / \gamma+i z}}{-1 / \gamma+i z} .
\end{aligned}
$$

Numerical computations of the Fourier transform $\hat{\varphi}_{3}(z)$, which are needed only when Condition A is not satisfied, are given in Section 4.1 of Nicolosi et al. (2018).

\section{B Proofs}

This section contains the proofs of Lemma 1 and Proposition 2.

Proof of Lemma 1 In the proof of this lemma we assume the following integrability condition:

$$
\mathbf{E}\left[\int_{0}^{T}\left(\zeta_{t}^{z} e^{A(t ; z)+B(t ; z) \pi_{t}+C(t ; z) \pi_{t}^{2}}\left(1+\pi_{t}\right)^{2}\right) d t\right]<\infty .
$$

We define the process

$$
I_{t}^{\mathbf{Q}}=I_{t}-\int_{0}^{t}\left(\sigma \beta-\pi_{s}\right) d s
$$


By Girsanov's Theorem this is a Q-brownian motion (see, e.g. Chap. 26 of Björk 2009). Then the $\mathbf{Q}$-dynamics of the filter $\pi_{t}$ is given by

$$
d \pi_{t}=\left(\lambda\left(\bar{X}-\pi_{t}\right)+\left(R_{t}+\rho \sigma_{X}\right)\left(\beta \sigma-\pi_{t}\right)\right) d t+\left(R_{t}+\rho \sigma_{X}\right) d I_{t}^{\mathbf{Q}}
$$

Using Ito's Lemma, we get that $\zeta_{t}=\xi_{t} Y^{\gamma}$ under $\mathbf{Q}$ has the following dynamics

$$
\begin{aligned}
\frac{d \zeta_{t}}{\zeta_{t}}= & \left(r(\gamma-1)+\frac{1}{2} \gamma(\gamma+1) \beta^{2} \sigma^{2}-(\gamma+1) \beta \sigma \pi_{t}+\pi_{t}^{2}\right) d t \\
& +\left(\gamma \beta \sigma-\pi_{t}\right) d I_{t}^{\mathbf{Q}} .
\end{aligned}
$$

Under condition (35), the process $H\left(t, \zeta_{t}, \pi_{t} ; z\right)$ is a $\left(\mathbb{F}^{S}, \mathbf{Q}\right)$-martingale (see, e.g. the discussion in Theorem 4 of Colaneri et al. 2020); then, equating the $d t$-term to zero leads to the partial differential equation (for simplicity we drop the arguments of the functions)

$$
\begin{aligned}
0= & \frac{\partial H}{\partial t}+\frac{\partial H}{\partial \zeta} \zeta\left(r(\gamma-1)+\frac{1}{2} \gamma(\gamma+1) \beta^{2} \sigma^{2}-(\gamma+1) \beta \sigma p+p^{2}\right) \\
& +\frac{\partial H}{\partial p}\left(\lambda(\bar{X}-p)+\left(R_{t}+\rho \sigma_{X}\right)(\beta \sigma-p)\right)+\frac{1}{2} \frac{\partial^{2} H}{\partial p^{2}}\left(R_{t}+\rho \sigma_{X}\right)^{2} \\
& +\frac{\partial^{2} H}{\partial \zeta \partial p} \zeta\left(R_{t}+\rho \sigma_{X}\right)(\gamma \beta \sigma-p)+\frac{1}{2} \frac{\partial^{2} H}{\partial \zeta^{2}} \zeta^{2}(\gamma \beta \sigma-p)^{2}
\end{aligned}
$$

with the boundary condition at time $T$

$$
H(T, \zeta, p ; z)=\zeta^{z}, \quad \zeta \in \mathbb{R}^{+}, p \in \mathbb{R}, z \in \mathbb{C}
$$

We use a similar approach as in the optimization problem under full information (see Nicolosi et al. 2018), and consider an exponential-polynomial ansatz of the type

$$
H(t, \zeta, p ; z)=\zeta^{z} e^{A(t ; z)+B(t ; z) p+\frac{1}{2} C(t ; z) p^{2}}
$$

where $A(t ; z), B(t ; z)$ and $C(t ; z)$ are deterministic functions. From the boundary condition (37) we get that

$$
A(T ; z)=0, \quad B(T ; z)=0, \quad C(T ; z)=0 .
$$

Moreover, substituting the partial derivatives of the function $H$ into (36) and imposing that coefficients of $p^{2}, p$ and the constant terms are equal to zero, we obtain the system 
of ordinary differential equations for $A(t ; z), B(t ; z)$ and $C(t ; z)$

$$
\begin{aligned}
\frac{\partial C}{\partial t}= & -\left(R_{t}+\rho \sigma_{X}\right)^{2} C^{2}+2\left(\lambda+(1+z)\left(R_{t}+\rho \sigma_{X}\right)\right) C-z(z+1) \\
\frac{\partial B}{\partial t}= & \left(\lambda+(z+1)\left(R_{t}+\rho \sigma_{X}\right)-\left(R_{t}+\rho \sigma_{X}\right)^{2} C\right) B \\
& -\left(\lambda \bar{X}+(1+z \gamma) \beta \sigma\left(R_{t}+\rho \sigma_{X}\right)\right) C+z(1+z \gamma) \beta \sigma \\
\frac{\partial A}{\partial t}= & z r(1-\gamma)-\frac{1}{2} z \gamma(1+z \gamma) \beta^{2} \sigma^{2}-\frac{1}{2}\left(R_{t}+\rho \sigma_{X}\right)^{2} B^{2} \\
& -\left(\lambda \bar{X}+(1+z \gamma) \beta \sigma\left(R_{t}+\rho \sigma_{X}\right)\right) B-\frac{1}{2}\left(R_{t}+\rho \sigma_{X}\right)^{2} C .
\end{aligned}
$$

Notice that this is a system of equations of Riccati type, with non-homogeneous coefficients.

Proof of Proposition 2 The proof of part ( $i$ ) follows the same lines of Nicolosi et al. (2018) (Nicolosi et al. 2018, Proposition 2.1). Here we summarize the idea. Since the market model under partial information is complete, after applying concavification we get that the relative final wealth $V_{T}$ is given by the formula (26) in Appendix A. Plugging the expression of $V_{T}$ into $V_{t}=\mathbf{E}^{\mathbf{Q}}\left[V_{T} \mid \mathcal{F}_{t}^{S}\right]$ and then using Fourier transform we can calculate the value at time $t$ of the optimal relative value. Note that here one needs to apply Fubini Theorem and change the order of integration. This operation is well defined, for instance, under conditions D1 and D2 of Proposition 2.7 in Eberlein et al. (2010), that, in our modelling framework are satisfied if $K_{1}<-1 / \gamma, K_{4}>$ $-1 / \gamma$, for any $K_{2}$ and $K_{3}$, and (20). The fact that $K_{2}$ and $K_{3}$ can be arbitrary is a consequence of the boundedness of function $\varphi_{2}$ and $\varphi_{3}$ in (28)-(29).

For part $(i i)$, we first determine the dynamics of $W_{t}^{\star}=Y_{t} V_{t}=Y_{t} V\left(t, \zeta_{t}, \pi_{t}\right)$ via Ito's product rule. Then comparing this equation with Eq. (14) provides the expression for $\theta_{t}$ in (22). Notice that the integrals in (21) are principal value integrals and the partial derivatives of the function $V$ in (22) can be computed from (21) by taking the derivative under the integral sign.

We remark that, if $\gamma>1$, since the function $f(w, y)$ is bounded, we have that

$$
\mathbf{E}\left[\frac{\left(W_{T} f\left(W_{T}, Y_{T}\right)\right)^{1-\gamma}}{1-\gamma}\right] \leq c \mathbf{E}\left[\frac{\left(W_{T}\right)^{1-\gamma}}{1-\gamma}\right]<\infty .
$$

If $\gamma \in(0,1)$ the value function is unbounded, hence the optimization problem may not be well posed. To exclude explosion, additional conditions must be imposed. In Proposition 2, condition (20) allows to apply Fourier transform, and then to characterize the relative wealth in terms of the conditional moment generating function $H(t, \zeta, p ; z)$ of $\ln \left(\zeta_{T}\right)$ and ensures that $V_{t}$ is finite as well. This condition is implied by existence of the solution of the system of Riccati ODEs for the functions $A, B, C$ up to time $T$ (equivalently, the explosion time of the ODEs is larger than the time horizon) for specific values of $z$ and some additional integrability, which permits to write equation (19). 


\section{Solutions to non-Homogeneous Riccati ODEs}

We discuss the solution of the system of non-homogeneous system of Riccati equations arising in the expression of the conditional moment generating function of $\ln \left(\zeta_{T}\right)$. Precisely, we show how to solve the system of Eqs. (40)-(41). Equation (42) can be computed by direct integration, and we do this numerically. Following, for instance, Brendle (2006) and Colaneri et al. (2020), it can be proved that the functions $B$ and $C$ satisfy

$$
\begin{aligned}
& C(t ; z)=\frac{C^{o}(t ; z)}{1+\frac{1}{z} C^{o}(t ; z) R_{t}} \\
& B(t ; z)=\frac{B^{o}(t ; z)}{1+\frac{1}{z} C^{o}(t ; z) R_{t}}
\end{aligned}
$$

for some functions $C^{o}(t ; z)$ and $B^{o}(t ; z)$ which solve the homogeneous system of Riccati equations below

$$
\begin{aligned}
\frac{\partial C^{o}}{\partial t}= & \left(\frac{1}{z}\left(1-\rho^{2}\right)-\rho^{2}\right) \sigma_{X}^{2} C^{o 2}+2\left(\lambda+(1+z) \rho \sigma_{X}\right) C^{o}-z(z+1) \\
\frac{\partial B^{o}}{\partial t}= & \left(\lambda+(z+1) \rho \sigma_{X}+\left(\frac{1}{z}\left(1-\rho^{2}\right)-\rho^{2}\right) \sigma_{X}^{2} C^{o}\right) B^{o} \\
& -\left(\lambda \bar{X}+(1+z \gamma) \beta \sigma \rho \sigma_{X}\right) C^{o}+z(1+z \gamma) \beta \sigma
\end{aligned}
$$

with boundary conditions

$$
B^{o}(T, z)=0, \quad C^{o}(T, z)=0
$$

Equations (43)-(44) have a solution in closed form ${ }^{6}$ see for instance Filipović (2009, Lemma 10.12).

6 Equations (43)-(44) are related to the conditional moment generating function of the process $\ln \left(\zeta_{T}\right)$, under full information. In fact, by Markovianity it holds that

$$
\widetilde{H}\left[t, \xi_{t}, X_{t} ; z\right]=\mathbf{E}^{Q}\left[\zeta_{T}^{z} \mid \mathcal{F}_{t}\right]
$$

Here using the ansatz $\widetilde{H}(t, \zeta, x ; z)=\zeta^{z} e^{A^{o}(t ; z)+B^{o}(t ; z) x+\frac{1}{2} C^{o}(t ; z) x^{2}}$ we get that $B^{o}(t ; z)$ and $C^{o}(t ; z)$ solve (43)-(44) with the boundary condition (45) and $A^{o}(t ; z)$ satisfies

$$
\frac{\partial A^{o}}{\partial t}=z r(1-\gamma)-\frac{1}{2} z \gamma(1+z \gamma) \beta^{2} \sigma^{2}-\left(\lambda \bar{X}+B^{o}(1+z \gamma) \beta \sigma \sigma_{X}\right)-\frac{1}{2} \sigma_{X}^{2}\left(C^{o}+B^{o 2}\right)
$$

with the boundary condition $A^{o}(T ; z)=0$. 

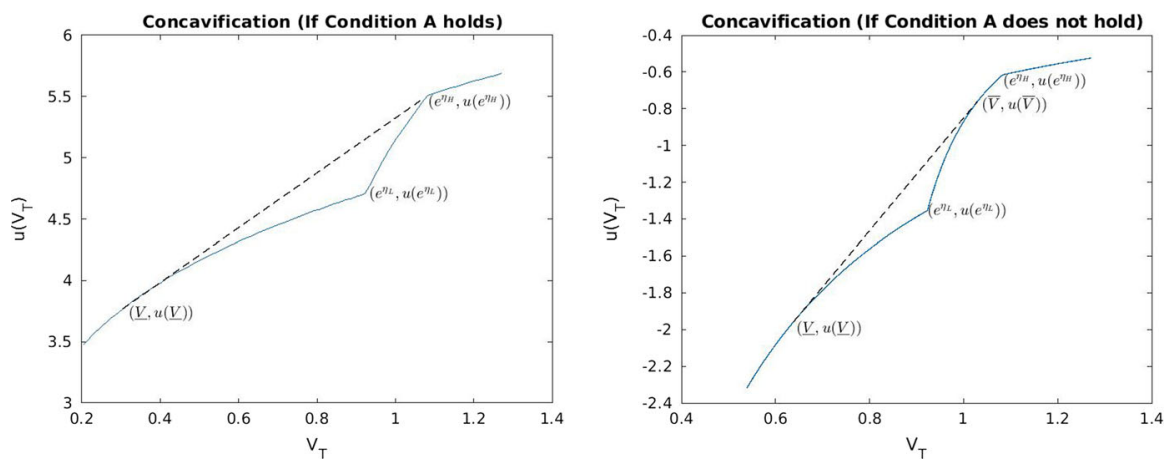

Fig. 5 This figure displays the concavified objective function for different levels of the relative terminal value $V_{T}=W_{T} / Y_{T}$. The solid line represents the original objective function and the superimposed dashed line is the concavification. The left panel corresponds to the case where Condition $A$ is satisfied, and the right panel the case where Condition $A$ is not satisfied

\section{The concavification}

In this section we provide a brief explanation on the concavification procedure proposed by Carpenter (2000). Let $u: D \subseteq \mathbb{R} \rightarrow \mathbb{R}$ be a function; the concavification of $u(x)$, if it exists, is the smallest concave function $\widetilde{u}(x)$, such that $\widetilde{u}(x) \geq u(x)$ for every $x \in D$. Consider now the objective function in our optimization problem $u\left(W_{T} f\left(W_{T}, Y_{T}\right)\right)=\frac{\left(W_{T} f\left(W_{T}, Y_{T}\right)\right)^{1-\gamma}}{1-\gamma}$, where the function $f$ is given in Eq. (7). We observe that the objective function can be represented in terms of the relative wealth $V_{T}=\frac{W_{T}}{Y_{T}}$ as $^{7}$

$$
u\left(V_{T}\right)=\left\{\begin{array}{ccc}
\frac{\left(V_{T} f_{L}\right)^{1-\gamma}}{1-\gamma} & \text { if } \quad V_{T}<e^{\eta_{L}} \\
\frac{\left(V_{T} f_{L}+\psi V_{T}\left(\log \left(V_{T}\right)-\eta_{L}\right)\right)^{1-\gamma}}{1-\gamma} & \text { if } e^{\eta_{L}} \leq V_{T}<e^{\eta_{H}} \\
\frac{\left(V_{T} f_{H}\right)^{1-\gamma}}{1-\gamma} & \text { if } \quad V_{T} \geq e^{\eta_{H}}
\end{array}\right.
$$

Due to the fact that incentives are nonlinear, the objective function is not globally concave in $V_{T}$, and hence we need to replace part of the original function with a chord between points $\underline{\mathrm{V}}$ and $\bar{V}$, called the concavification points. If the original function is smooth in $\underline{\mathrm{V}}$ and $\bar{V}$, then the slope of the chord equals the slope of the function. For the objective function $u$ considered in this paper, the concavification is represented in Fig. 5. The solid line represents the original objective function and the dashed line its concavification. The left and right panels show the case where Condition A is met and the case when it is not satisfied, respectively. In particular, we see from the left panel that the objective function is not smooth at point $\bar{V}$ and hence this concavification point coincides with an angle point.

The importance of Condition A is also related to the representation of the solution of the optimization problem: if it is satisfied, an analytical expression for the optimal

\footnotetext{
$\overline{7}$ This representation comes from a combination of a change of variable and the change of measure $\frac{d \mathbf{Q}}{d \mathbf{P}}$.
} 
value and the optimal strategies can be derived; otherwise, optimal value, and hence optimal strategy, can only be computed numerically (cf. Appendix A).

\section{References}

Altay S, Colaneri K, Eksi Z (2018) Pairs trading under drift uncertainty and risk penalization. Int J Theor Appl Finance 21(7):56

Altay S, Colaneri K, Eksi Z (2020) Optimal converge trading with unobservable pricing errors. Ann Oper Res. https://doi.org/10.1007/s10479-020-03647-z

Barucci E, Marazzina D (2015) Risk seeking, nonconvex remuneration and regime switching. Int J Theor Appl Finance 18(2):56. https://doi.org/10.1142/S0219024915500090

Barucci E, Marazzina D (2016) Asset management, high water mark and flow of funds. Oper Res Lett 44(5):607-611

Barucci E, Marazzina D, Mastrogiacomo E (2019) Optimal investment strategies with a minimum performance constraint. Ann Oper Res 56:1-25

Basak S, Pavlova A (2013) Asset prices and institutional investors. Am Econ Rev 103(5):1728-1758

Basak S, Pavlova A, Shapiro A (2007) Optimal asset allocation and risk shifting in money management. Rev Financ Stud 20(5):1583-1621

Basak S, Pavlova A, Shapiro A (2008) Offsetting the implicit incentives: benefits of benchmarking in money management. J Bank Finance 32:1883-1893

Björk T (2009) Arbitrage theory in continuous. Oxford University Press

Björk T, Davis MH, Landen C (2010) Optimal investment under partial information. Math Methods Oper Res 71(2):371-399

Brendle S (2006) Portfolio selection under incomplete information. Stoch Process Appl 116(5):701-723

Brennan M (1998) The role of learning in dynamic portfolio decisions. Eur Financ Rev 1:295-306

Buraschi A, Kosowski R, Sritrakul W (2014) Incentives and endogenous risk taking: a structural view on hedge fund alphas. J Finance 69(6):2819-2870

Carpenter JN (2000) Does option compensation increase managerial risk appetite? J Finance 55(5):23112331

Ceci C, Colaneri K (2012) Nonlinear filtering for jump diffusion observations. Adv Appl Probab 44(3):678701

Ceci C, Colaneri K (2014) The Zakai equation of nonlinear filtering for jump-diffusion observations: existence and uniqueness. Appl Math Optim 69(1):47-82

Ceci C, Colaneri K (2017) Recent advances in nonlinear filtering with a financial application to derivatives hedging under incomplete information. Bayesian Inference 5:325-348

Chen HL, Pennacchi GG (2009) Does prior performance affect a mutual fund's choice of risk? Theory and further empirical evidence. J Financ Quant Anal 56:745-775

Chevalier J, Ellison G (1997) Risk taking by mutual funds as a response to incentives. J Polit Econ 105(6):1167-1200

Colaneri K, Herzel S, Nicolosi M (2021) The value of knowing the market price of risk. Ann Oper Res 299:101-131. https://doi.org/10.1007/s10479-020-03596-7

Cox JC, Huang CF (1989) Optimal consumptions and portfolio policies when asset prices follow a diffusion process. J Econ Theory 49:33-83

Cuoco D, Kaniel R (2011) Equilibrium prices in the presence of delegated portfolio management. J Financ Econ 101(2):264-296

Eberlein E, Glau K, Papapantoleon A (2010) Analysis of Fourier transform valuation formulas and applications. Appl Math Finance 17(3):211-240

Filipovic D (2009) Term-structure models. A graduate course. Springer

Fleming WH, Pardoux É (1982) Optimal control for partially observed diffusions. SIAM J Control Optim 20(2):261-285

Hata H, Sheu S (2018) An optimal consumption and investment problem with partial information. Adv Appl Probab 50:131-153

Herzel S, Nicolosi M (2019) Optimal strategies with option compensation under mean reverting returns or volatilities. Comput Manag Sci 16:47-69. https://doi.org/10.1007/s10287-017-0296-3 
Huang JC, Wei KD, Yan H (2012) Investor learning and mutual fund flows. In: AFA 2012 Chicago meetings paper

Lakner P (1995) Utility maximization with partial information. Stoch Process Appl 56(2):247-273

Lakner P (1998) Optimal trading strategy for an investor: the case of partial information. Stoch Process Appl 76(1):77-97

Lan Y, Wang N, Yang J (2013) The economics of hedge funds. J Financ Econ 110(2):300-323

Lee S, Papanicolaou A (2016) Pairs trading of two assets with uncertainty in co-integration's level of mean reversion. Int J Theor Appl Finance 19(8):1650054

Lindensjo K (2016) Optimal investment and consumption under partial information. Math Methods Oper Res 83:87-107

Lipster RS, Shiryaev AN (2001) Statistics of random processes. Springer, Berlin

Ma L, Tang Y, Gomez J (2019) Portfolio manager compensation in the US mutual fund industry. J Finance 76(2):587-638

Merton RC (1971) Optimum consumption and portfolio rules in a continuous-time model. J Econ Theory 3:373-413

Nicolosi M (2018) Optimal strategy for a fund manager with option compensation. Decis Econ Financ 41:1-17. https://doi.org/10.1007/s10203-017-0204-X

Nicolosi M, Angelini F, Herzel S (2018) Portfolio management with benchmark related incentives under mean reverting processes. Ann Oper Res 266(1-2):373-394

Xia Y (2001) Learning about predictability: the effects of parameter uncertainty on dynamic asset allocation. J Finance 56:205-246

Publisher's Note Springer Nature remains neutral with regard to jurisdictional claims in published maps and institutional affiliations. 